\title{
Origine culturelle et sociale de la violence à l'école
} Les dimensions culturelles des relations et des conduites agressives pendant l'enfance

\section{Cultural and social origins of school violence}

The cultural dimensions of relationships and aggressive behaviour during childhood

\section{Origen cultural y social de la violencia en la escuela} Las dimensiones culturales de las relaciones y
comportamientos agresivos durante la infancia

\section{Monique Allès-Jardel et Barry Schneider}

Volume 32, numéro 1, printemps 2004

La violence en milieu scolaire

URI : https://id.erudit.org/iderudit/1079124ar

DOI : https://doi.org/10.7202/1079124ar

Aller au sommaire du numéro

\section{Éditeur(s)}

Association canadienne d'éducation de langue française

\section{ISSN}

0849-1089 (imprimé)

1916-8659 (numérique)

\section{Découvrir la revue}

\section{Citer cet article}

Allès-Jardel, M. \& Schneider, B. (2004). Origine culturelle et sociale de la violence à l'école : les dimensions culturelles des relations et des conduites agressives pendant l'enfance. Éducation et francophonie, 32(1), 224-244. https://doi.org/10.7202/1079124ar
Résumé de l'article

Comprendre les phénomènes de violence entre les jeunes en particulier à l'école est devenu un défi majeur pour la plupart des sociétés occidentales. Cette compréhension passe par l'observation des conduites agressives et des déterminants de celles-ci. Du biologique au social, des caractéristiques individuelles aux caractéristiques environnementales, les causes invoquées sont multiples, et par conséquent tout autant les théories psychosociologiques qui les sous-tendent. Dans ce texte, nous nous interrogerons sur les origines sociales et surtout culturelles de ces comportements. En effet, les caractéristiques culturelles semblent affecter les processus de socialisation, les relations interpersonnelles et moduler les manifestations des conduites agressives ainsi que leurs représentations. Les comparaisons interculturelles des comportements sociaux et « antisociaux » devraient, en effet, aider à identifier si il existe des processus de socialisation spécifiques à chaque société pouvant être aux origines de l'agressivité. Les travaux portant sur ces processus dans cette perspective comparative devraient permettre de trouver des liens existants entre les caractéristiques d'une société et le comportement social des individus qui la composent. Ce texte présente un ensemble de recherches concernant ce type d'influence en mettant l'accent sur la complexité et la composition multiculturelle de plusieurs sociétés. Nous proposons ici une synthèse de cette recension d'écrits. Nous présenterons tout d'abord des recherches permettant de comprendre comment la culture influence les relations sociales entre enfants et nous centrerons ensuite notre propos sur l'étude comparative de l'agressivité dans différentes cultures. Nous soulignerons, en outre, les défis méthodologiques reliés à la comparaison interculturelle des comportements violents pendant l'enfance.

Tous droits réservés $@$ Association canadienne d’éducation de langue française, Ce document est protégé par la loi sur le droit d’auteur. L’utilisation des 2004 services d’Érudit (y compris la reproduction) est assujettie à sa politique d'utilisation que vous pouvez consulter en ligne.

https://apropos.erudit.org/fr/usagers/politique-dutilisation/ 


\title{
Origine culturelle et sociale de la violence à l'école : les dimensions culturelles des relations et des conduites agressives pendant l'enfance
}

\author{
Monique Allès-Jardel
}

UFR STAPS, Université Paul-Sabatier, France

\section{Barry Schneider}

UFR STAPS, Université Paul-Sabatier, France

\section{RÉSUMÉ}

Comprendre les phénomènes de violence entre les jeunes en particulier à l'école est devenu un défi majeur pour la plupart des sociétés occidentales. Cette compréhension passe par l'observation des conduites agressives et des déterminants de cellesci. Du biologique au social, des caractéristiques individuelles aux caractéristiques environnementales, les causes invoquées sont multiples, et par conséquent tout autant les théories psychosociologiques qui les sous-tendent. Dans ce texte, nous nous interrogerons sur les origines sociales et surtout culturelles de ces comportements. En effet, les caractéristiques culturelles semblent affecter les processus de socialisation, les relations interpersonnelles et moduler les manifestations des conduites agressives ainsi que leurs représentations. Les comparaisons interculturelles des comportements sociaux et " antisociaux " devraient, en effet, aider à identifier si il existe des processus de socialisation spécifiques à chaque société pouvant être aux origines de l'agressivité.

Les travaux portant sur ces processus dans cette perspective comparative devraient permettre de trouver des liens existants entre les caractéristiques d'une société et le comportement social des individus qui la composent. Ce texte présente 
Origine culturelle et sociale de la violence à l'école:

les dimensions culturelles des relations et des conduites agressives pendant l'enfance

un ensemble de recherches concernant ce type d'influence en mettant l'accent sur la complexité et la composition multiculturelle de plusieurs sociétés. Nous proposons ici une synthèse de cette recension d'écrits. Nous présenterons tout d'abord des recherches permettant de comprendre comment la culture influence les relations sociales entre enfants et nous centrerons ensuite notre propos sur l'étude comparative de l'agressivité dans différentes cultures. Nous soulignerons, en outre, les défis méthodologiques reliés à la comparaison interculturelle des comportements violents pendant l'enfance.

\section{ABSTRACT}

Cultural and social origins of school violence: the cultural dimensions of relationships and aggressive behaviour during childhood

Monique Allès-Jardel and Barry Schneider

Understanding the phenomenon of violence among young people in particular at school, has become a major challenge in the majority of western societies. This understanding comes from the observation of aggressive behaviours and their underlying factors. From biological to social, from individual to environmental, the causes put forward are numerous, as are the psychosocial theories that support them. In this article, we explore the social and, above all, cultural origins of these behaviours. Cultural factures appear to affect the socialization process, interpersonal relationships and to influence the manifestation of aggressive behaviours as well as the forms they take. Intercultural comparisons of social and "antisocial behaviours" should help identify whether there are specific socialization processes in each society that could be at the origin of aggressiveness.

Studies of these processes in this comparative perspective should reveal existing links between the characteristics of society and the social behaviours of the individuals within that society. This article presents a body of research concerning this type of influence while stressing the complexity and the multicultural composition of several societies. We are presenting a synthesis of this body of work. First we present research that provides an understanding of how culture influences social relationships among children. We then focus on a comparative study of aggressiveness in different cultures. Furthermore, we emphasize the methodological challenges related to intercultural comparisons of violent behaviour during childhood. 
Origine culturelle et sociale de la violence à l'école:

les dimensions culturelles des relations et des conduites agressives pendant l'enfance

RESUMEN

Origen cultural y social de la violencia en la escuela: las dimensiones culturales de las relaciones y comportamientos agresivos durante la infancia

Monique Allès-Jardel y Barry Schneider

Comprender el fenómeno de la violencia entre los jóvenes particularmente en la escuela se ha convertido en un desafío de talla para la mayor parte de sociedades occidentales. La comprensión requiere la observación de las conductas agresivas y de sus determinantes. De lo biológico a lo social, de las características individuales a las características del medio ambiente, las causas invocadas son múltiples, lo mismo que las teorías psicológicas sobra las que se apoyan. En este texto, nos interrogamos sobre los orígenes sociales y culturales de dichos comportamientos. En efecto, las características culturales parecen afectar los procesos de socialización, las relaciones interpersonales y modular las manifestaciones de los conductas agresivas así como sus representaciones. Las comparaciones interculturales de los comportamientos sociales y 'anti-sociales' deberían en efecto facilitar la identificación de procesos de socialización específicos en cada sociedad de los cuales se origine la agresividad.

Los trabajos que abordan dichos procesos desde una perspectiva comparativa deberían facilitar la identificación de los lazos que existen entre las características de una sociedad y el comportamiento social de los individuos que la integran. Este texto presenta un conjunto de investigaciones sobre dicho tipo de influencia, subrayando la complejidad y la composición multicultural de varias sociedades. Proponemos aquí una síntesis de la revista de la literatura. Primero presentamos las investigaciones que nos permiten comprender cómo la cultura ejerce una influencia sobre las relaciones sociales entres los niños para enseguida centrarnos en el estudio comparativo de la agresividad en las diferentes culturas. Enfatizaremos, además, los retos metodológicos que conlleva la comparación intercultural de los comportamientos violentos durante la infancia.

\section{Introduction}

Les sociétés occidentales sont de plus en plus confrontées à la violence chez les jusqu'aux phénomènes de violence à l'école et dans les quartiers. jeunes et à des conduites relevant de l'agressivité, depuis les incivilités de plus en plus nombreuses jusqu'aux phénomènes de violence à l'école et dans les quartiers. Ce phénomène tend à se manifester de plus en plus jeune chez l'enfant, parfois dès l'âge de l'école maternelle dans le cadre scolaire ou non. Comprendre les raisons de ces comportements est devenu en conséquence un défi majeur. Cette compréhension passe par l'observation des conduites agressives et des déterminants de celles-ci. Les recherches récentes démontrent que l'étude de ces comportements est particulière- 
Origine culturelle et sociale de la violence à l'école:

les dimensions culturelles des relations et des conduites agressives pendant l'enfance

Les caractéristiques culturelles semblent affecter les processus de socialisation, les relations interpersonnelles et moduler les manifestations des conduites agressives ainsi que leurs représentations. ment complexe autant lorsqu'il s'agit d'en faire une analyse descriptive que d'en rechercher les processus sous jacents. Du biologique au social, des caractéristiques individuelles aux caractéristiques environnementales, les causes invoquées sont multiples, et par conséquent tout autant les théories psychosociologiques qui les sous-tendent. Mais de fait, la socialisation consiste en général à apprendre et à intérioriser les modèles culturels. Dans ce texte, nous nous interrogerons par conséquent sur les origines sociales et surtout culturelles de ces comportements. En effet, les caractéristiques culturelles semblent affecter les processus de socialisation, les relations interpersonnelles et moduler les manifestations des conduites agressives ainsi que leurs représentations.

Les comparaisons interculturelles des comportements sociaux et «antisociaux" devraient nous permettre de déterminer les processus de socialisation et les origines de l'agressivité. Il s'agit ici non pas d' identifier les cultures à risque mais de travailler à saisir quelles relations existent au sein même des cultures et entre elles et en quoi ces relations affectent-elles les comportements? Les travaux portant sur les processus de socialisation dans cette perspective comparative devraient permettre de trouver les liens entre les caractéristiques d'une société et le comportement social des individus qui la composent. Ces tentatives se sont souvent avérées positives comme le prouvent plusieurs études mentionnées plus loin. Ce texte est une recension d'écrits, il présente donc un ensemble de travaux concernant ce type d'influence en mettant l'accent sur la complexité et la composition multiculturelle de plusieurs sociétés. Nous proposons ici une synthèse. Nous présenterons tout d'abord des recherches permettant de comprendre comment la culture influence les relations sociales entre enfants et nous centrerons ensuite notre propos sur l'étude comparative de l'agressivité dans différentes cultures. Nous soulignerons, en outre, en préambule les difficultés d'une telle entreprise tenant au sujet lui même. L'étude des comportements violents pendant l'enfance peut, en effet, soulever des questions éthiques mais elle représente en même temps un véritable défi méthodologique qui est inhérent à la comparaison interculturelle. Ces points seront abordés dans la discussion.

\section{Perspectives interculturelles pour les recherches sur les processus de socialisation et les comportements agressifs}

Un ensemble de recherches interculturelles a permis tout d'abord de démontrer que certains aspects spécifiques des relations sociales chez l'enfant ne sont pas très différents d'une culture à l'autre. Ces études permettent de saisir les aspects universels du comportement social. Les résultats nous apprennent qu'en fait les enfants de cultures différentes utilisent un petit nombre de scénarios d'interactions sociales (Whiting \& Edwards, 1988). Cependant, les différences de culture peuvent affecter les représentations et la façon dont l'enfant exprime ses compétences sociales. Dans la plupart des sociétés, ils expriment le désir d'acquérir des connaissances sur euxmêmes, sur autrui et sur les interactions sociales afin de devenir des individus compétents socialement (Edwards, 1986). Cependant l'appartenance culturelle peut d'une 
Origine culturelle et sociale de la violence à l'école:

les dimensions culturelles des relations et des conduites agressives pendant l'enfance

part influencer la manière dont l'enfant manifeste et exprime ce besoin important qui est de devenir habile socialement et d'autre part elle peut aussi affecter les différentes étapes auxquelles surviennent les événements signifiants pour la socialisation.

Dans la littérature scientifique, la recherche sur les liens entre la culture et les relations entre pairs se partage en deux approches principales. La première compare certaines particularités comme la religion, l'économie, ou l'idéologie dans une culture donnée avec les mêmes caractéristiques dans d'autres cultures; les cultures étant considérées comme distinctes et séparées. Une utilisation pertinente de cette approche consiste à utiliser un échantillonnage important de cultures différentes variant sur une dimension spécifique. Par exemple, une recherche peut proposer d'étudier le niveau d'agressivité générale dans des sociétés de plusieurs pays pour pouvoir apprécier comment celle-ci se manifeste chez les enfants et entraîne leur adhésion. Mais cette recherche interculturelle comparative représente un véritable défi et exige une bonne communication entre les chercheurs impliqués dans les différents pays. Les ressources matérielles et humaines doivent êtres en outre équivalents dans chacun des sites où sont collectées les données. Les études les plus précises et détaillées ont été le plus souvent réalisées sur les relations interpersonnelles entre adultes, mais ce type de recherche est de plus en plus utilisé pour faire des investigations à propos des relations sociales chez les enfants.

Un deuxième type d'approche examine les relations entre les individus de différentes sub-cultures coexistant dans une même culture majoritaire. Ce qui est le cas dans de nombreux pays occidentaux, qui accueille de nombreux immigrés. Au cours du vingtième siècle, des individus fuyant les problèmes économiques et politiques ont cherché un refuge en Amérique du Nord, en Europe Occidentale, en Australie, ou dans d'autres sociétés occidentales. De plus, la création d'unions comme l'Union Européenne et le groupe nord américain de libres échanges a provoqué une augmentation des mouvements de population. Ces changements sociaux et politiques fournissent de nombreuses occasions pour étudier des phénomènes comme les amitiés interethniques et pour mesurer l'influence de la culture sur les choix affiliatifs des enfants. De nombreuses recherches ont été réalisées sur ce sujet. Cet intérêt s'explique en partie, par exemple, aux Etats-Unis par l'importance que les médias ont donné aux événements concernant le morcellement racial dans les écoles américaines et la violence qui règne entre les jeunes de différentes origines culturelles en GrandeBretagne, en France et en Allemagne.

\section{Individualisme/collectivisme}

Une classification fondamentale utilisée pour caractériser les cultures est de savoir si elles sont individualistes ou collectiviste (Hofstede, 1983). Le collectivisme prône l'importance de l'unité du groupe par rapport à l'autonomie individuelle généralement associée à l'individualisme. Dans les cultures collectivistes, les personnes travaillent pour atteindre des objectifs communs et assument des responsabilités collectives pour le bien-être du groupe. Ceci se traduit souvent par un partage des responsabilités pour la garde et l'éducation des enfants. 
Origine culturelle et sociale de la violence à l'école:

les dimensions culturelles des relations et des conduites agressives pendant l'enfance

Les pays anglophones; le Canada, la Grande-Bretagne, les Etats-Unis et l'Australie sont les sociétés considérées comme étant les plus individualistes (Hofstede, 1983). Dans ces nations, les enfants sont élevés dans un système de convictions qui valorise l'autonomie personnelle et l'individualisme. Dans les cultures individualistes, les croyances concernant autrui ne sont pas forcément partagées par tous. Dans la plupart des pays occidentaux, des travaux comme ceux de Madsen \& Shapira, (1970) ont montré que les habitants de zones urbaines présentent habituellement plus de comportements individualistes que ceux des zones rurales. L'immigration tout comme l'exode rural par ailleurs implique fréquemment, des changements vers des formes de sociétés et de relations sociales plus individualistes (Triandis \& coll., 1988).

En fait, il ne faudrait pas penser actuellement l'individualisme versus le collectivisme en termes d'opposition mais plutôt comme un continuum. Mais ce qui semble caractériser la plupart des sociétés actuellement de par le monde c'est une évolution vers plus d'individualisme. Les valeurs de certaines sociétés traditionnelles plus collectivistes du fait de l'urbanisation en particulier, tendant à disparaître.

Ainsi des travaux récents ont montré qu'il peut y avoir des variations très importantes à l'intérieur d'une même société sur cette dimension individualisme/collectivisme. C'est le cas, par exemple des Coréens de culture collectiviste qui travaillent en ville et qui sont connus pour êtres beaucoup plus individualistes que leurs concitoyens (Cha, 1994). En effet, il est courant qu'une société présente à la fois des caractéristiques individualistes et collectivistes. Par exemple, les Indiens vivant en Inde et à l'étranger font preuve d'idéaux collectivistes dans leurs relations familiales et d'orientations individualistes dans les relations interpersonnelles et les activités économiques (Sinha \& Tripathi, 1994). Plusieurs recherches ont mis en évidence des différences importantes entre des cultures qui sont habituellement considérées comme étant individualistes. Les Nord Américains attachent une grande importance à la poursuite d'intérêts individuels, tandis que les Européens Occidentaux valorisent l'égalitarisme et l'équilibre dans les relations, tout en valorisant aussi l'autonomie individuelle (Schwartz \& Ros, 1995, pp. 111-112). Dans un débat sur les interactions dynamiques entre les forces collectivistes et individualistes à l'intérieur d'une société, Philipsen (1987) a mis l'accent sur le fait que les cultures sont en constante évolution et ne restent pas toujours fixés en ces termes d'opposition entre collectivisme et d'individualisme. Il a aussi insisté sur le fait que pour appréhender complètement une culture, les recherches doivent comprendre non seulement en quoi elles sont collectivistes ou individualistes, mais doivent aussi saisir les forces en œuvre à l'intérieur de la culture qui la rendent individualiste ou collectiviste. En effet, des forces concurrentes règnent dans de nombreuses sociétés, tendant vers un plus grand individualisme ou un plus grand collectivisme.

L'identité culturelle est généralement renforcée chez les membres d'un groupe culturel donné quand le nombre de contacts entre différents groupes culturels est important; ceci s'applique typiquement aux sociétés multiculturelles (McGuire, McGuire, Child \& Fujioka, 1978), particulièrement parmi les groupes minoritaires (Hewstone, Bond \& Wan, 1983; Hofman, 1985). Plusieurs recherches ont étudié l'identité cultu- 
Origine culturelle et sociale de la violence à l'école:

les dimensions culturelles des relations et des conduites agressives pendant l'enfance

relle des enfants dans de nombreuses sociétés. Les résultats des études dans lesquelles une minorité collectiviste est entourée par une culture majoritaire indiquent deux tendances opposées. En effet, s'ils sont entourés par une majorité, les parents de minorité s'attacheront à leur propre identité culturelle et protégeront leurs enfants de l'assimilation en inculquant leurs valeurs culturelles. Cependant, différentes solutions peuvent êtres adoptés, par lesquelles les parents apprennent à leurs enfants les compétences nécessaires pour s'intégrer dans la culture majoritaire (Ogbu, 1981) sans pour autant renier les valeurs de la culture d'origine. Cette tendance peut impliquer d'adopter des styles éducatifs différents afin de transmettre les capacités nécessaires pour progresser dans une société individualiste.

Gudykunst et Ting-Toomey (1988) soutiennent que l'individualisme et le collec-

L'individualisme et le collectivisme ne sont pas les seules dimensions qui influencent les relations sociales dans une société mais ce sont celles qui apparaissent comme ayant le plus d'effets sur l'organisation sociale. une société mais ce sont celles qui apparaissent comme ayant le plus d'effets sur l'organisation sociale.

Il y a quelques années, il était courant de trouver des articles, dans des revues (essentiellement anglophones) consacrés au développement, rapportant les résultats de comparaisons portant sur les comportements d'enfants de différents pays, sans donner d'explication circonstanciée sur les raisons de celles-ci. Cependant, plus récemment, nous constatons un intérêt croissant dans le cadre des recherches entreprises pour un approfondissement théorique concernant par exemple, Les explications sur les effets du collectivisme ou de l'individualisme sur les comportements de compétition des enfants.

Nous pouvons nous attendre à ce que ces cultures dans lesquelles sont fortement valorisés les besoins et les désirs individuels favorisent les intérêts personnels. (c'est-à-dire des cultures mettant l'accent sur l'individualisme, l'autonomie, et la compétition) accepteront mieux les comportements agressifs que les cultures dans lesquelles l'accent est mis sur les objectifs du groupe, le respect de la collectivité et de l'environnement (c'est-à-dire les cultures ne favorisant pas l'individualisme mais au contraire l'harmonie, le conservatisme et l'intégration). Les membres des cultures individualistes auront beaucoup plus volontiers recours à l'agressivité parce que celle-ci peut faciliter la réalisation de leurs objectifs individuels. L'usage de l'agressivité dans les cultures dans lesquelles les individus se conçoivent comme faisant partie du groupe est moins favorisé, parce qu'un tel comportement détruit l'harmonie du groupe et n'est pas bénéfique à la collectivité.

Les anthropologistes ont noté que dans beaucoup de sociétés où l'agressivité s'observe rarement, les individus sont socialisés pour développer une conception personnelle de dépendance à l'égard du groupe, où le collectif plutôt que l'individualité est encouragé [Fry, 1999a, 1999b; Robarchek \& Robarchek, 1998]. Dans ces sociétés non-agressives, une grande valeur est attribuée à l'interdépendance entre les membres du groupe et les comportements de partage, d'aide, de générosité sont fortement encouragés. Ceci contraste encore plus avec les cultures agressives où les valeurs sont placées dans l'individualisme et l'autonomie et où leurs membres se considèrent comme indépendants les uns des autres et ne comptent que sur euxmêmes [Fry, 1999a, 1999b; Robarchek \& Robarchek, 1998]. 
Origine culturelle et sociale de la violence à l'école:

les dimensions culturelles des relations et des conduites agressives pendant l'enfance

Toutes les études interculturelles présentant un intérêt ne pourront êtres présentés dans ce chapitre. En conséquence, nous proposerons dans la suite de ce texte une sélection de recherches comparative interculturelles représentative des études sur les relations entre pairs.

\section{Exemples de recherches comparatives sur les processus de socialisation dans des sociétés collectivistes et individualistes}

\section{Premier exemple : comparaison entre les sociétés individualistes occidentales et une société collectiviste comme la Chine Caractéristiques de la société chinoise}

Des études structurées ont permis aux scientifiques de faire un parallèle entre les principes fondamentaux de la société chinoise et les patterns de relations observés dans les relations entre pairs. Nous n'en présenterons ici que quelques exemples. De fait la Chine est très différente de l'Amérique du nord ou de l'Europe occidentale sur cette dimension, elle occupe l'extrémité du spectre en terme de collectivisme (Triandis, 1990). Ce contraste est souvent utilisé pour rendre compte des différences de relations sociales entre ces deux sociétés. Le collectivisme inhérent à la culture chinoise est considéré comme fournissant un modèle d'organisation du comportement social, les interactions entre individus devant se révéler en accord avec les besoins et les attentes du groupe. En effet, selon Yang (1981), les chinois anticipent les réactions des autres et modulent leur comportement en conséquence. Nous pouvons rapprocher ces considérations générales des observations de Toupin (1980) selon lesquelles cette société réprime l'expression directe des sentiments et des émotions. En conséquence les conflits verbaux et les confrontations directes vont à l'encontre de la conception chinoise de la tenue et des manières à adopter en publique («face» en anglais) (Chiu, Tsang \& Yang, 1988). La résolution des conflits se fait par la médiation et la négociation.

\section{Étude comparative des relations sociales des enfants chinois et des enfants} occidentaux

Dans leur étude portant sur l'observation et la comparaison de la coopération et des conflits chez des enfants chinois et canadiens de 5 ans, Orlick, Zhou et Partington (1990) ont mis en évidence une interdépendance mutuelle chez les jeunes enfants chinois. Ils ont trouvé que ces derniers s'engagent plus dans des comportements pro-sociaux, de type : aide et partage, que les enfants canadiens. Les petits Canadiens font preuve de moins de comportements coopératifs et $78 \%$ de leurs comportements impliquent des conflits. Ces chercheurs (1990) ont suggéré que ces différences interculturelles sont dues à des différences dans la socialisation des enfants dans les deux populations. Malgré, des différences évidentes dans les valeurs fondamentales de ces deux sociétés, il existe cependant des ressemblances importantes dans les 
Origine culturelle et sociale de la violence à l'école:

les dimensions culturelles des relations et des conduites agressives pendant l'enfance

relations entre pairs des enfants chinois et canadiens. Chen, Rubin et Li (1994) ont trouvé que le comportement pro-social et l'agression sont corrélés avec l'ajustement social et inversement; ces résultats sont analogues à ceux d'échantillons occidentaux.

\section{Deuxième exemple : comparaison d'enfants latino-américains et d'enfants de sociétés occidentales les plus individualistes}

Les études réalisées en Amérique Latine constituent une autre contribution consistante pour les recherches sur les relations entre pairs. L'Amérique Latine, comme les sociétés de l'Asie de l'Est, est une société fortement collectiviste, la famille élargie est un élément primordial de la vie quotidienne et joue un rôle de conseil et de soutien. Cependant, pour plusieurs raisons, les comparaisons entre enfants d'Amérique du Sud et d'Amérique du Nord ou d'Europe occidentale ne fournissent pas de données aussi contrastées que les études réalisées en Asie de l'Est. La plupart des pays d'Amérique Latine partagent de nombreuses croyances religieuses avec l'Amérique du Nord et l'Europe Occidentale et des points communs en ce qui concerne leur histoire culturelle. Les Pays d'Amérique Latine présentent beaucoup moins de différences entre les différentes cultures les composants qu'aux Etats-Unis ou au Canada, mais bon nombre d'entre eux sont plus hétérogènes que la Chine, la Corée ou le Japon. Les classes sociales défavorisées prédominent en Amérique Latine, mais la différence est considérable entre les conditions de vie entre des classes défavorisées et les classes moyennes et les milieux favorisés.

Les comparaisons du comportement d'enfants mexicains et américains ont été d'une grande importance dans la compréhension sur la façon dont la compétitivité d'une société se reflète dans les rivalités entre enfants. Dans une étude classique, Madsen a mis en évidence que les enfants mexicains font preuve d'un grand nombre de comportements coopératifs. Dans une autre expérience, Kagan et Madsen (1971) ont testé des enfants anglo-américains et des enfants mexicains dans un dispositif destiné à récompenser les interactions coopératives, la compétition au contraire n'entraînait pas de récompenses. Ils ont trouvé que parmi les paires constituées d'enfants de 7-9 ans, ce sont les enfants mexicains qui étaient les plus coopératifs, les anglo-américains l'étaient le moins et les Mexicains américains se situaient à un niveau intermédiaire. Dans une autre étude, les résultats indiquaient que les enfants anglo-américains répondaient de manière plus conflictuelle dans des situations sociales antagonistes que les enfants mexicains (Kagan \& Madsen, 1972).

Dans une étude similaire, les résultats montraient qu'en utilisant un jeu de «marbel-pull», il y avait une augmentation de compétitions inadaptées par rapport à l'âge chez les enfants anglo-américains (Madsen, 1971). Kagan et Madsen (1971) ont ainsi émis l'hypothèse selon laquelle les niveaux de compétition et de coopération sont influencés par les orientations plus ou moins forte en direction du groupe. En se plaçant dans une perspective génétique de type piagetien, Madsen (1971) suggérait que l'égocentrisme et le désir de se mettre en avant est si fort chez les enfants anglo-américains plus âgés cela peut entraver l'interaction coopérative qui permet d'obtenir des gratifications. Et ceci même et si ils font preuve de capacités de résolu- 
Origine culturelle et sociale de la violence à l'école:

les dimensions culturelles des relations et des conduites agressives pendant l'enfance

tion de problèmes et sont donc aptes à reconnaître que la compétition peut s'avérer être le comportement le plus adapté dans certaines circonstances.

Après ces quelques exemples contrastés démontrant un certain nombre de liens pouvant effectivement existés entre la culture et les comportements sociaux des enfants, nous allons maintenant centrer davantage encore notre propos sur les comparaisons des conduites agressives des enfants dans les différentes sociétés.

\section{Les dimensions culturelles de l'agressivité pendant I'enfance : quelques autres exemples}

Les communautés Zapotèques de la région d'Oaxaca au Mexique offre également une occasion intéressante d'examiner le lien entre l'agressivité des adultes et les comportements agressifs des enfants envers leurs pairs. Les Zapotèques, qui subsistent essentiellement grâce à l'agriculture, sont organisés en petites communautés séparées. Le taux d'agressivité varie de manière importante dans ces différentes communautés. Par exemple dans les 24 communautés Zapotèques étudiées jusqu'ici, le taux annuel d'homicide est compris entre 4 et 123 personnes sur 100000 . Il n'est pas surprenant en conséquence, que les attitudes envers la violence varient tout autant. Dans les communautés les moins agressives, les bagarres et les combats même sous forme de jeux sont considérés comme préjudiciables. Cependant, les communautés les plus violentes sont caractérisées par des jeux brutaux et des bagarres nombreuses entre adultes, aussi bien que par le recours aux punitions corporelles envers les enfants.

Fry (1988) a étudié les comportements de 24 enfants de 5 à 8 ans issus de deux communautés Zapotèques présentant différents degrés de violence. Les observations ont porté sur des combats réels, des bagarres sous forme de jeu et des comportements d'intimidation. Les résultats indiquent que le taux de jeux agressifs était deux fois plus élevé dans la communauté de San Andres que dans celle de La Paz considérée comme la moins violente ( 6.9 contre 3.7 incidents par heure). Les recherches ont montré aussi que les taux d'agressions réels étaient en fait moins importants

L’agressivité varie à travers les cultures et même à l'intérieure d'une même culture dans des communautés différentes. dans les deux communautés. Néanmoins, les enfants de San Andres faisaient preuve d'un taux d'agressions plus élevé que ceux de La Paz (0.7 incidents contre 0.39 incident par heure). Les enfants plus âgés de San Andres s'engageaient plus fréquemment que les plus jeunes dans des bagarres réelles, tandis que dans la communauté de la Paz, le taux de bagarres était plus bas chez les enfants les plus âgés. Les corrélations entre les bagarres réelles ou simulées se sont avérées faibles, ce qui montre que la plupart des enfants tend à s'engager aussi bien dans l'une ou l'autre forme de ces manifestations agressives. Les résultats de cette étude démontrent que l'agressivité varie à travers les cultures et même à l'intérieure d'une même culture dans des communautés différentes. Les objectifs développementaux peuvent êtres modifiés par le contexte culturel. Ces résultats renforcent aussi l'affirmation de Smith (1989) selon laquelle les enfants se préparent à leur rôle d'adulte au sein de leur communauté par les jeux de compétition et des bagarres. 
Origine culturelle et sociale de la violence à l'école:

les dimensions culturelles des relations et des conduites agressives pendant l'enfance

Les études italiennes sont également d'excellentes sources d'informations illustrant les différences culturelles vis-à-vis de l'agressivité, particulièrement pour ce qui est des attentes différentes concernant les filles et les garçons (Schneider, 1971). La société traditionnelle italienne tolère et même encourage dans une certaine mesure l'agressivité chez les hommes mais pas chez les femmes. Nous disposons également de données similaires, provenant d'études effectuées dans des centres urbains importants essentiellement dans le nord et le centre de l'Italie, concernant les relations entre le comportement et le statut sociométrique chez les enfants préscolaires italiens (Fonzi, Tomada \& Cuicci, 1994) de moyenne section (Attili, Vermigli \& Schneider, 1997). Ces études réalisées sur de larges échantillons ont utilisé des mesures similaires à celles qui sont employées dans de nombreuses études américaines. Les résultats indiquaient qu'en Italie le modèle de ce comportement était corrélés significativement avec l'acceptation par les pairs de manière similaire à ce qui a été mis en évidence dans de nombreux autres pays et dans des études nord améri-

Il existe de nombreux invariants culturels dans l'acceptation des pairs au sein des groupes d'enfants, malgré la présence probables de différences interculturelles. caines réalisées auprès d'enfants préscolaires et d'école élémentaire. Mais, à la différence, des études conduites dans le centre de l'Italie et dans le nord, les jeunes du sud de l'Italie semblent concevoir que certaines formes d'agressivité entre pairs sont acceptables socialement (Casiglia, LoCoco \& Zappulla, 1998). Ces données combinées avec celles d'autres pays étayent la conclusion selon laquelle il existe de nombreux invariants culturels dans l'acceptation des pairs au sein des groupes d'enfants, malgré la présence probables de différences interculturelles. Ainsi, des similitudes interculturelles constatés indiquent également, par exemple que les comportements agressifs et perturbateurs sont liés avec le rejet par les pairs, tandis que les comportements pro-sociaux sont associés à l'acceptation par les pairs.

Dans leur étude menée parmi les enfants de petites villes du centre de l'Italie, Tomada et Schneider (1997) ont tenté de répliquer les résultats de Crick et Grotpeter (1995) qui indiquaient que les garçons américains faisaient preuve d'un taux d'agressivité direct et d'un niveau d'agressivité relationnelle plus important que les filles. Comme il en a été débattu précédemment, l'agressivité directe fait référence aux agressions physiques et verbales d'hostilité visant directement une autre personne. L'agressivité relationnelle fait souvent référence à des formes insidieuses d'agression dans les relations interpersonnelles, comme le fait de diffuser une rumeur, ou l'exclusion d'un enfant du groupe de jeux (Crick \& Grotpeter, 1995; Lagerspetz, Björkqvist \& Peltonen, 1988). L'agressivité manifeste est courante dans les cours de récréation en Italie. Genta et ces collaborateurs (1996) ont trouvé que les enfants italiens qui fréquentent l'école élémentaire du centre de l'Italie font preuve d'un taux significativement plus élevé de comportements de menaces et d'intimidations qu'en Norvège, en Angleterre, en Espagne et au Japon.

La manifestation de l'agressivité chez les garçons italiens est le plus souvent considérée comme normale et elle est même encouragée chez les adolescents dans les villages traditionnels italiens comme un témoignage s'apparentant à une prouesse sexuelle et une préparation à leur futur rôle patriarcal. Par contre l'agressivité extériorisée est désapprouvée chez les filles (Maraspini, 1968; Schneider,1971). Tomada et Schneider (1997) ont analysé les descriptions faites par des professeurs et des élèves 
Origine culturelle et sociale de la violence à l'école:

les dimensions culturelles des relations et des conduites agressives pendant l'enfance

d'écoles élémentaires italiennes en ce qui concerne les comportements agressifs et pro-sociaux. Ils ont trouvé suite à leurs différentes études que les filles italiennes n'expriment pas un taux d'agressivité relationnelle plus important que les garçons, ces derniers ont des taux d'agressivité manifeste et d'agressivité relationnelle plus forts que ceux des filles. Si l'agressivité relationnelle n'était pas prise en compte, on n'en arriverait à la conclusion absurde selon laquelle l'agressivité serait relativement absente chez les filles italiennes. Des études comparant de grands échantillons d'un grand nombre de pays permettraient de comprendre l'intérêt d'étudier l'agressivité relationnelle dans d'autres sociétés.

Ainsi, un projet portant sur six sociétés différentes (Whiting \& Edwards,1988; Whiting \& Whiting, 1975) a entrepris d'identifier les différences culturelles présentes dès le début du développement social de l'enfant. Les recherches avaient recours à l'observation directe des mères et de leurs enfants âgés de 2 à 10 ans en Inde, à Okinawa, aux Philippines, au Mexique, au Kenya et aux Etats-Unis. Les interactions mère-enfants et les relations sociales entre enfants ont été ainsi codés. Les informations sur chacune des cultures étaient bien documentées, ce qui ajoutait encore à la valeur de ce travail. Cette étude est restituée en détail dans deux volumes qui incluent en outre des descriptions des conditions de la vie quotidienne, des ressources, des pratiques éducatives parentales, du réseau de soutien social et du type de responsabilité donné aux enfants dans chaque pays. Ce projet a mis en évidence des différences culturelles importantes entre les sociétés étudiées. Par exemple, les enfants d'âge scolaire dans les cultures ayant un système scolaire organisé ont plus de contacts avec leurs pairs, surtout avec ceux du même sexe.

Dans une autre étude, à Nyansago au Kenya, où un seul enfant sur les 22 sujets de la recherche allait à l'école, les résultats indiquaient le plus faible taux de contact entre pairs. Dans cette étude, les enfants ont rarement fait preuve de comportements agressifs, ceci est particulièrement vrai pour les filles. Le taux le plus important de comportement agressif a été attribué aux enfants de Khalapur, en Inde, culture qui pratique les châtiments corporels envers les enfants. Les enfants de Mixteca, une communauté indienne du Mexique, faisaient preuve eux aussi d'un niveau important de conduites agressives. Dans cette communauté, les niveaux d'agressivité des adultes est particulièrement élevé. Les garçons qui appartiennent à des cultures encourageant la ségrégation sexuelle et la compétition scolaire, sont souvent très bagarreurs. Ceux qui font le plus preuve de comportements protecteurs et éducatifs envers les pairs de même sexe vivent généralement dans des cultures où il est demandé aux enfants qu'ils aident leurs frères et sœurs.

Osterman et ses collègues (1994) ont réalisé une étude interculturelle de l'agression et de la "victimisation » chez des enfants appartenant à cinq groupes ethniques différents. Les données ont été collecté auprès d'enfants de 8 ans en Turquie, Finlande (parlant le suédois et le Finnois), à Chicago aux Etats-Unis (des caucasiens et les noirs américains) et à Varsovie en Pologne. En utilisant l'échelle d'agressivité directe et indirecte (Björkqvist, Lagerspetz \& Osterman, 1992), les chercheurs ont trouvé que les enfants noirs américains avaient le niveau d'agressivité le plus fort qu'elle soit citée par eux-mêmes ou par leurs pairs. Les auteurs attribuent les résul- 
Origine culturelle et sociale de la violence à l'école:

les dimensions culturelles des relations et des conduites agressives pendant l'enfance

tats constatés à la violence qui règne dans la communauté des enfants noirs américains de Chicago, mais à partir de cette constatation nous ne savons pas véritablement quelle est le lien de cause à effet. Quoique les scores des garçons ne différent pas significativement d'une culture à l'autre, par contre ceux des filles présentent des variations significatives. Par exemple les filles polonaises indiquaient un niveau d'agression important dans leur propre évaluation et qui était confirmé par les réponses données par les pairs. Un tel niveau d'agression peut s'expliquer par la tradition d'opposition aux pouvoirs étrangers qui a longtemps existé en Pologne (Osterman \& coll., 1994).

Farver et ses collaborateurs (1997) ont aussi trouvé un grand niveau d'agressivité parmi les enfants américains. Dans leur étude interculturelle réalisée en Suisse, en Allemagne, en Indonésie et aux Etats-Unis, 40 enfants de 4 ans dans chaque pays devaient créer des histoires en utilisant des jouets à connotation agressive ou non. L'analyse de contenu des scénarios des jeunes enfants américains montraient un plus grand nombre de situations, de mots et de personnages agressifs que celles des enfants des autres pays. Les histoires, des petits Américains présentaient, de plus, des situations beaucoup plus agressives que celles des autres enfants. Fraver et al. suggèrent que le taux de crimes et de violence élevé aux Etats-Unis influence le développement de l'agression chez les enfants. Ils insistent aussi sur le fait que dans la société américaine hautement individualiste, la socialisation des enfants accentue la compétition, l'indépendance, l'indifférence émotionnelle par rapport aux besoins des autres. La plus grande différence de niveau d'agressivité se trouvait entre les enfants américains et les enfants indonésiens. Ceci est probablement le reflet des différences culturelles dans les pratiques éducatives et dans la tolérance vis-à-vis des comportements agressifs. Farser et ses collaborateurs font référence aux résultats de Triandis (1990) selon lesquels la société indonésienne est caractérisée par le collectivisme et un certain mépris pour l'agressivité. Comparés aux enfants américains, les enfants indonésiens ne sont pas encouragés à devenir indépendants et compétitifs, mais la collectivité leur apprend à valoriser l'harmonie, la coopération et l'importance de la communauté et de la famille. Cette étude met en évidence comment l'agressivité exprimée dans des récits d'enfants, peut refléter les caractéristiques culturelles.

Huesmann, Lagerspetz et Eron (1984) ont réalisé une étude interculturelle afin de déterminer s'il était possible de trouver des différences culturelles dans les corrélations entre le comportement agressif d'enfants américains et Finlandais d'âges scolaires et le fait de regarder la télévision. Ces enfants ont été testés et interviewés pendant trois ans. Les résultats de cette étude longitudinale suggèrent que le fait de regarder des programmes violents à la télévision est lié à l'agressivité et peut prédire une augmentation des niveaux d'agressivité chez les filles américaines et les garçons américains et finlandais. L'importance de la relation entre les deux dépend à la fois de l'intensité de la violence des émissions regardées et du temps passé devant le petit écran. Pour les enfants américains, ces deux facteurs auraient une répercussion d'importance équivalente, bien que le contenu des programmes de télévision finlandais soit beaucoup plus violent. La corrélation entre télévision et agressivité était d'autant plus accentuée chez les garçons, qu'ils s'identifiaient aux personnages. La violence 
Origine culturelle et sociale de la violence à l'école:

les dimensions culturelles des relations et des conduites agressives pendant l'enfance

des programmes à la télévision finlandaise est contrôlée bien que la plupart des émissions violentes soit importés. Huesmann, Lagerspetz et Eron (1984) postulaient que les enfants finlandais ne s'identifient pas à des émissions violentes ou pensent qu'elles sont réelles. En fait les résultats des études portant sur les relations entre les programmes télévisés et l'expression de l'agressivité sont souvent contradictoires. En fait des recherches plus récentes tendraient à montrer que ce sont les enfants les plus agressifs qui regardent ce genre d'émissions.

Dans une autre étude, Eron et Huesmann (1987) ont examiné les relations entre le fait de regarder la télévision, l'évaluation de l'agressivité par les pairs et la popularité parmi des jeunes israéliens vivant dans un kibboutz et d'autres vivant en ville. Ils ont aussi fait des collectes de données auprès d'enfants australiens, américains, finlandais et polonais. Il y a de nombreux points communs entre les différents échantillons étudiés. L'agressivité évaluée par les pairs est restée stable sur une période de trois ans et elle était négativement corrélée avec la popularité dans toutes les populations bien qu'il a pu être constaté une variabilité interculturelle dans le degré par lequel le fait de regarder la télévision induit l'agressivité. Cela était particulièrement évident dans le fait de regarder des programmes violents. La corrélation la plus faible se trouvait chez les enfants du kibboutz, ce qui peut être imputé à la régulation effectuée par les personnes en charge de la garde des enfants lorsqu'ils regardent la télévision. En effet, celles-ci discutent de l'implication de la violence à la fin des émissions. Il se pourrait que l'impact de la violence à la télévision sur les enfants soit affaiblis par la nature collectiviste des communautés des kibboutz et les discussions qui suivent qui offrent un accompagnement aux enfants sur le sens à donner à ces programmes.

Des différences interculturelles peuvent également affecter le niveau de tolérance vis-à-vis des comportements atypiques et ces différents seuils peuvent déterminer comment l'adulte réagit aux comportements agressifs des enfants. Schneider, Attili, Vermigli et Younger (1997) ont comparé les croyances de parents canadiens et italiens à propos de l'agressivité et du retrait social. Ils ont émis l'hypothèse selon laquelle les mères italiennes considéreraient l'agressivité comme plus inquiétante et les mères canadiennes auraient des réponses émotionnelles plus fortes par rapport au retrait social. Ils ont aussi étudié la répartition des mères qui ont des enfants identifiés comme retirés ou agressifs. Les résultats indiquent que les mères canadiennes considéraient le retrait social comme plus problématique que les mères italiennes. Toutefois, les deux échantillons indiquaient que l'agressivité était considérée comme plus alarmante que le retrait social. Les chercheurs ont rencontré des différences intéressantes dues au facteur sexe, l'intensité des réactions émotionnelles des mères italiennes par rapport au retrait social de leur fille était moins importante que la réaction à ce comportement s'il se manifestait chez les garçons, cette différence due au sexe n'a pas été retrouvée dans l'échantillon canadien. Une grande proportion de mères italiennes pense que les problèmes de comportement de l'enfant dépendent de facteurs psychologiques stables et internes comme les traits de personnalité. Par contre, les mères canadiennes aspiraient à croire que les comportements des enfants pouvaient êtres facilement changés. Cette différence peut être reliée aux croyances qui ont cours dans les villages italiens concernant le rôle du destin et de la chance 
Origine culturelle et sociale de la violence à l'école:

les dimensions culturelles des relations et des conduites agressives pendant l'enfance

dans l'évolution future des enfants (Maraspini, 1968). Il n'est pas considéré en général que le comportement puisse être modifié par les attitudes éducatives. Au contraire, les mères canadiennes semblent plus persuadées que les interventions parentales et le style éducatif peuvent modifier le comportement de l'enfant. En conséquence, cette étude peut aider à comprendre comment les caractéristiques culturelles peuvent influencer les interprétations que les parents font du comportement de leurs enfants aussi bien que leurs niveaux de tolérance vis-à-vis du retrait social et à l'agressivité.

Weisz, Suwanlert, Chaiyasit et Weiss (1988) ont aussi étudié les seuils de tolérance au comportement sur-contrôlé (ex: timidité, retrait) et au comportement souscontrôlé (ex : agressivité), dans une recherche conduite en Thaïlande, en Jamaïque et aux Etats-Unis. La majorité des Thaïlandais souscrivent à la doctrine du bouddhisme thaïlandais. L'étude consistait en la présentation à des parents et enseignants américains et thaillandais de deux images, chacune d'elle illustrant un exemple du pattern de comportement étudié. Ils devaient alors estimer le degré de gravité du comportement et dire s'il était peu comme cela ou pas. De plus, les parents devaient évaluer leur niveau de préoccupation par rapport au comportement s'ils étaient le parent du protagoniste ou l'enseignant. Ils avaient aussi à apprécier la probabilité d'un changement du comportement et trouver ce qui l'avait suscité et ce qu'ils devaient faire. Les résultats indiquent que les sujets thaïlandais évaluaient les comportements souscontrôlé et sur-contrôlé moins négativement que les sujets américains. En comparaison, par rapport aux participants américains les Thaïlandais jugeaient aussi ces patterns de comportement comme moins inquiétants pour un enseignant ou un parent, et plus probable de s'améliorer tout seul. Les psychologues thaïlandais et américains rapportaient des niveaux égaux de préoccupation à propos des problèmes de comportement. Le niveau de préoccupation des psychologues était plus important que celui des parents et des professeurs thaïlandais, mais plus bas que ceux du groupe de sujets américains. Les Thaïlandais attribuaient plus souvent les comportements surcontrôlés et sous-contrôlés à une éducation défectueuse que ne le faisaient les Américains qui tendaient à attribuer ces comportements à des facteurs de stress environnementaux, comme une vie familiale et interpersonnelle instable et des conflits personnels. Dans les deux cultures, le comportement sous-contrôlé semblait plus préoccupant que le comportement sur-contrôlé.

French, Setiono et Eddy (1999) ont étudié l'agressivité chez les enfants en Indonésie. La société javanaise indonésienne est extrêmement collectiviste (Hofstede, 1991) et met l'accent sur la coopération et l'harmonie dans les relations (Koentjaraningrat, 1985). En utilisant le paradigme individualisme/collectivisme, French, Setiono et Eddy ont émis l'hypothèse selon laquelle l'agressivité serait associée à un statut sociométrique négatif et ce dans les deux pays, mais cela serait plus particulièrement prononcé en Indonésie. Ils prédisaient aussi que le retrait social serait associé à un statut social élevé chez les enfants américains, mais non chez les enfants indonésiens. Les résultats témoignent de l'association attendue entre l'agressivité et un statut sociométrique négatif. French, Setiono et Eddy suggèrent que ce résultat peut être un reflet de perturbations dans les relations pendant l'enfance (voir Patterson, 1982), plus que comme un phénomène individuel. Les conclusions mon- 
Origine culturelle et sociale de la violence à l'école:

les dimensions culturelles des relations et des conduites agressives pendant l'enfance

trent aussi des similitudes interculturelles en termes de relation entre l'acceptation sociométrique et l'amitié réciproque. Cependant dans l'échantillon américain, les enfants qui étaient agressifs avaient un groupe de pairs et des amis alors que ce n'étaient pas le cas pour les enfants indonésiens. En Indonésie, les enfants agressifs ont rarement des amis. Les résultats selon lesquels les enfants indonésiens avaient moins d'amitiés réciproques peuvent refléter l'idéologie collectiviste, les groupes de pairs harmonieux sont communément estimé sur une petite quantité d'amitié intime (Noesjirwan, 1978).

Les diverses études présentées permettent de cerner les relations pouvant exister entre les différents types de culture et l'expression et manifestations de l'agressivité ches les enfants et les adolescents sans que le constat des liens de cause à effet soit toujours évident à établir de même que les explications qui peuvent être fournis.

\section{Conclusion : des défis que représentent la recherche interculturelle}

Le sujet de cette recension de travaux analysés à partir des publications disponibles représente un sujet critique d'autant plus qu'il contient aussi sa part de considérations éthiques. Il importe bien évidemment de bien distinguer dans ce compte-rendu entre populations étudiées dans leur pays d'origine et populations étudiées dans leurs pays d'adoption. Les premières composent véritablement avec les valeurs culturelles propres à leur milieu. Les secondes ajoutent une donnée, à savoir quelle interaction éco-systémique leur situation d'immigrants ajoute à ces valeurs et leur mise en œuvre. Nous nous sommes efforcés de rapporter ici le plus fidèlement possibles le contenu de ces différentes recherches et approches. Toutes les études interculturelles présentant un intérêt pour la compréhension de la manifestation des comportements d'agressivité chez les enfants n'ont pas pu êtres passés en revue dans ce texte de synthèse.

Les travaux impliquant les enfants ne sont pas toujours faciles à mener surtout dans certaines sociétés et dans certains pays, la recherche interculturelle représente un véritable défi supplémentaire qui exige une bonne communication entre les chercheurs impliqués dans les différents pays. Dans l'idéal il serait nécessaire que les ressources matérielles et humaines doivent êtres équivalents et aussi disponibles dans chacun des sites où sont collectées les données. Mais c'est évidemment toute la difficulté des études interculturelles. Les études les plus détaillées ont été le plus souvent réalisées sur les relations interpersonnelles entre adultes, mais ce type de comparaison et de plus en plus utilisé pour faire des recherches sur des enfants comme il est démontré dans cet exposé. Cette approche étant fondamentale pour tenter de cerner et de comprendre les origines sociales et culturelles des comportements agressifs et de la violence chez les jeunes.

Les études rapportées témoignent des capacités des chercheurs à surmonter certaines des difficultés et les nombreux obstacles inhérents à la comparaison de données issues de différentes sociétés. Passer en revue les résultats a permis de voir 
Origine culturelle et sociale de la violence à l'école:

les dimensions culturelles des relations et des conduites agressives pendant l'enfance

que la comparaison des relations entre pairs dans différents pays n'est pas simple. En premier lieu, ces recherches impliquent nécessairement une information approfondie sur chacun des pays comparés. De telles connaissances peuvent permettre d'établir, tout d'abord, que la question qui va être étudiée a une plus ou moins grande importance selon les sociétés. Dans la plupart des pays, il existe en effet des distinctions spécifiques concernant l'ethnicité, les statuts socio-économiques ou les différences entre sexes, qui permettent d'expliquer les différences observables dans les valeurs affichées et les comportements sociaux qui s'expriment de façon plus ou moins contrastés selon le pays. Une difficulté méthodologique supplémentaire réside dans la traduction et la nécessaire adaptation des instruments utilisés sans perdre certaines des informations originelles. En outre, les participants de différentes sociétés peuvent ressentir différemment le fait d'être impliqué dans une recherche, notre expérience montre par exemple qu'il est plus difficile de pénétrer dans les familles françaises qu'en Amérique du Nord. Certains peuvent considérer cela comme une source de nouveauté et de fierté, d'autres peuvent considérer cela comme une intrusion dans leur vie privée. Les chercheurs et les étudiants issus de divers pays peuvent également présenter des différences au niveau de la formation à la recherche.

Aucune des recherches existantes sur les différences culturelles dans les relations entre pairs chez l'enfant ne peut résoudre tous ces problèmes. Néanmoins, les fondements des recherches à venir indiquent des différences importantes au niveau des relations entre pairs dans les différentes sociétés, dans des secteurs-clé comme l'acceptation par les pairs des enfants timides et l'importance de la compétition entre les enfants. D'autres recherches servent d'autres objectifs de la recherche interculturelle : par exemple pour démontrer comment les enfants présentent des caractéristiques communes et des comportements similaires dans les différentes sociétés. Le rejet presque universel par les pairs des enfants qui font preuve d'agressivité malintentionnée est en est un exemple.

De fait, nous espérons que cet exposé très documenté en décrivant comment les caractéristiques d'une société et d'une culture peuvent affecter les processus de socialisation en général et les conduites agressives plus particulièrement, puisque telle était notre propos, aura permis de faire un pas dans la compréhension de ces phénomènes d'agressivité et de violence par la prise en compte des spécificités culturelles. Même si il est difficile de présenter une vision complète et intégrée des différents éléments, ceci devrait être pris en compte dans les études ultérieures et dans les éventuels programmes de prévention en direction des jeunes. 


\section{Références bibliographiques}

Argyle, Micheal. (1983). The Psychology of interpersonal behaviour. London, Routledge.

Attili, G., Vermigli, P. et Schneider, B. H. (1997). « Peer acceptance and friendship patterns among Italian elementary-school children within a cross-cultural perspective ». International Journal of Behavioural Development vol. XXI, pp. 277-298.

Björkqvist, K., Largerspetz, K. M. J. et Osterman, K. (1992). The direct and indirect aggression scales. Vasa, Finland: Abo Akademi University, Departement des Sciences Sociales.

Casiglia, A. C., Lococo, A. et Zappulla, C. (1998). «Aspects of social reputation and peer relationships in Italian children : a cross-cultural perspective». Developmental Psychology vol. XXXIV, pp. 723-730.

Cha, J. H. (1994). "Aspects of individualism and collectivism in Korea», dans Individualism and collectivism : theory, methods, and applications, sous la direction de U. Kim, H. C. Triandis, C. Kagitcibasi, S. Choi et G. Yoon : Thousand Oaks, Californie : Sage, pp.157-174.

Chen, X., Rubin, K. H. et Li, Z. Y. (juillet 1994). «Social functioning and adjustment in Chinese Children : a longitudinal study». Papier presenté à la réunion de la Societé Internationale pour les Études du Developpement Comportementale. Amsterdam, Netherlands.

Chui, C. Y., Tsang, S. C. et Yang, C. F. (1988). «The role of face situation and attitudinal antecedents in chinese consumer complaint behaviour». The Journal of Social Psychology vol. CXXVIII, pp. 173-180.

Crick, N. R. et Grotpeter, J. K. (1995). «Relational aggression, gender, and socialpsychological adjustment». Child Development vol. LXVI, pp. 710-722.

Edwards, C. P. (1986). Promoting social and moral development in young children: creative approaches for the classroom. New York: Teachers College Press.

Eron, L. D. et Huesmann, L. R. (1987). «The stability of aggressive behaviour in cross-national comparison", dans Growth and progress in cross-cultural psychology, sous la direction de C. Kagitcibasi. Lisse, Netherlands : Swets, pp. 207-217.

Farver, J. M., Welles-Nyström, B, Frosch, D. L., Wibarti, S. et Hoppe-Graff, S. (1997). "Toy stories: aggression in children's narratives in the United States, Sweden, Germany and Indonesia ». Journal of Cross-Cultural Psychology vol. XXVIII, pp. 393-420.

Fonzi, A., Tomada, G. et Ciucci, E. (1994). «Uso di indici informativi nell'interazione tra bambini del nido». Eta evolutiva vol. XLVII, pp. 5-13. 
Origine culturelle et sociale de la violence à l'école:

les dimensions culturelles des relations et des conduites agressives pendant l'enfance

French, D. C., Setonio, K. et Eddy, J. M. (1999). «Bootstrapping through the cultural comparison minefield : childhood social status and friendship in the United States and Indonesia ", dans Relationships as developmental contexts. The Minnesota symposia on child psychology vol. XXX. Mahwah N. J. : Lawrence Erlbaum Associates, pp. 109-131.

Fry, D. P. (1999a): Peaceful societies. In Kurtz LR (ed): Æncyclopedia of Violence, Peace, and Conflict. Vol. 2. @ San Diego: Academic Press, pp. 719-733.

Fry, D. P (1999b): Aggression and altruism. In Kurtz LR (ed): AEncyclopedia of Violence, Peace, and Conflict. Vol. 1. @ San Diego: Academic Press, 17-33.

Fry, D. P. (1988). «Intercommunity differences in aggression among Zapotec children ». Child Development vol. LIX, pp. 1008-1019.

Genta, M. L., Menesini, E., Fonzi, A., Constable, A. et Smith, P. K. (1996). «Bullies and victims in schools in central and southern Italy». European Journal of Psychology of Education vol. XI, pp. 97-110.

Gundykunst, W. B. et Ting-Toomey, S. (1988). Culture and interpersonal communication. Newbury Park CA: Sage.

Hewstone, M., Bond, M. H. et Wan, K. (1983). «Social facts and social attributions : the explanation of intergroup differences in Hong Kong». Social Cognition vol. II, pp. 142-157.

Hofman, P. (1985). «Apperçus de la prise en charge educative». Neuropsychiatrie de l'Enfance et de l'Adolescence vol. XXXIII, pp. 37-42.

Hofstede, G. (1983). «Dimensions of national cultures in fifty countries and three regions ", dans Explications in cross-cultural psychology, sous la direction de J. Deregowski, S. Dzuirawiec \& R. Annis. Lisse, Netherlands : Swets and Zeitlinger, pp. 335-355.

Hofstede, G. (1991). Cultures and organization: software on the mind. London : McGraw-Hill.

Huesmann, L. R., Lagerspetz, K. et Eron, L. D. (1984). «Intervening variables in the TV violence-aggression relation: evidence from two countries». Developmental Psychology vol. XX, pp. 746-775.

Kagan, S. et Madsen, M. C. (1971). «Co-operation and competition of Mexican, Mexican-American, and Anglo-American children of two ages under four instructional sets ». Developmental Psychology vol. V, pp. 32-39.

Kagan, S. et Madsen, M. C. (1972). «Experimental analysis of cooperation and competition of Anglo-American and Mexican children ». Developmental Psychology vol. VI, pp. 49-59.

Koentjaraningrat, R. M. (1985). Javanese culture. New York: Oxford University Press. 
Origine culturelle et sociale de la violence à l'école:

les dimensions culturelles des relations et des conduites agressives pendant l'enfance

Lagerspetz, K. M., Björkqvist, K. et Peltonen, T. (1988). « Is indirect aggression typical of females? Gender differences in aggressiveness in 11- to 12-year-old children ». Aggressive Behaviour vol. XIV, pp. 403-414.

Madsen, M. C. (1971). «Developmental and cross-cultural differences in the co-operative and competitive behaviour of young children ». Journal of Cross-Cultural Psychology vol. II, pp. 365-371.

Madsen, M. C. et Shapira, A. (1970). "Co-operative and competitive behaviour of urban Afro-American, Anglo-American, Mexican-American, and Mexican village children ». Developmental Psychology vol. III, pp. 16-20.

Maraspini, A. J. (1968). The study of an Italian village. Paris : Mouton.

Mcguire, W. J., Mcguire, C. V., Child, P. et Fujioka, T. (1978). «Salience of ethnicity in the spontaneous self-concept as a function of one's ethnic distinctiveness in the social environment ». Journal of Personality and Social Psychology vol. XXXVI, pp. 511-520.

Noesjirwan, J. (1978). «A rule-based analysis of cultural differences in social behaviour : Indonesia and Australia». International Journal of Psychology vol. XIII, pp. 305-316.

Ogbu, J. U. (1981). «Origins of human competence : a cultural-ecological perspective». Child Development vol. LII, pp. 413-429.

Orlick, T., Zhou, Q. Y. et Partington, J. (1990). «Co-operation and conflict within Chinese and Canadian kindergarten settings ». Canadian Journal of Behavioural Science vol. XXII, pp. 20-25.

Osterman, K., Björkqvist, K., Lagerspetz, K. M. J., Kaukiainen, A., Huesmann, L. R. et Franczek, A. (1994). "Peer and self-estimated aggression and victimization in 8-year-old children from 5 ethnic groups ». Aggressive Behaviour vol. XX, pp. 411-428.

Patterson, G. R. (1982). A social learning approach vol. III, Coercive family processes. Eugene, OR : Castalia.

Philipsen, G. (1987). «The prospect for cultural communication », dans Communication theory: Eastern and Western perspectives, sous la direction de D. Kincaid. New York: Academic Press, pp. 245-254.

Robarchek CA, Robarchek CJ. (1998). Reciprocities and realities: World views, peacefulness, and violence among Semai and Waorani. Aggressive Behavior 24:123-133.

Schneider, B. H., Attili, G., Vermigli, P. et Younger, Alastair. (1997). «A comparison of middle-class English-Canadian and Italian mothers' beliefs about children's peer-directed aggression and social withdrawal ». International Journal of Behavioural Development vol. XXI, pp. 133-154. 
Origine culturelle et sociale de la violence à l'école:

les dimensions culturelles des relations et des conduites agressives pendant l'enfance

Schneider, J. (1971). «On vigilance and virgins : honour, shame, and access to resources in Mediterranean societies ». Ethnology vol. X, pp. 1-23.

Schwartz, S. H. et Ros, M. (1995). «Value priorities is West European nations : a cross-cultural perspective", dans Studies in psychology in honor of Solomon Kugelmass, sous la direction de G. Ben-Shakar \& A. Lieblich. Jerusalem : Magnes Press, pp. 322-347.

Sinha, J. B. P. et Tripathi, R. C. (1994). «Individualism in a collectivist culture», dans Individualism and collectivism : theory, methods, and applications, sous la direction de U. Kim, H. C. Triandis, C. Kagitcibasi, S. C. Choi and G. Yoon. Thousand Oaks, CA: Sage, pp. 123-136.

Smith, P. K. (1989). "The role of rough and tumble play in the development of social competence : theoretical perspectives and empirical evidence», dans Social competence in developmental perspectives, sous la direction de Barry H. Schneider, G. Attili, J. Nadel \& R. P. Weissberg. Dordrecht : Kluwer, pp. 239-255.

Tomada, G. et Schneider, B; H. (1997). «Relational aggression, gender, and peer acceptance: Invariance across culture, stability over time, and concordance among informants ». Developmental Psychology vol. XXXIII, pp. 601-609.

Toupin, E. S. W. A. (1980). «Counselling Asians : psychotherapy in the context of racism and Asian-American history ». American Journal of Orthopsychiatry vol. L, pp. 76-86.

Triandis, H. C. (1990). "Cross-cultural studies of individualism and collectivism», dans Nebraska symposium on motivation vol. XXXVII, sous la direction de R. A. Dienstbier (ed. de la série) \& J. J. Berman (ed. du vol.). Lincoln : University of Nebraska Press, pp. 41-133.

Triandis, H. C., Bontempo, R., Villareal, M. J., Asai, M. et Lucca, N. (1988). «Individualism and collectivism: cross-cultural perspectives on self-ingroup relationships ». Journal of Personality and Social Psychology vol. LII, pp. 323-338.

Weisz, J. R., Suwalert, S., Chaiyasit, W. et Weiss, B. (1988). «Thai and American perspectives on over and undercontrolled child behaviour problems : exploring the threshold model among parents, teachers, and psychologists ". Journal of Counselling and Clinical Psychology vol. LVI, pp. 601-609.

Whiting, B. B. et Edwards, C. P. (1988). Children of different worlds : the formation of social behaviour. Cambridge, MA: Harvard University Press.

Whiting, B. B. et Whiting, J. W. M. (1975). Children of six cultures: a psychocultural analysis. Cambridge, MA: Harvard University Press.

Yang, K. S. (1981). «Social orientation and individual modernity among Chinese students in Taiwan ». Journal of Social Psychology vol. CXIII, pp. 159-170. 